Crônicas da ATUALIdAde do direito internacional .................................................. 2

I. Dossiê Temático: Direito Transnacional .........................................................15

EDITORIAL: O Direito Transnacional - Circulação de normas e relações jurídicas transnacionais .......16 Priscila Pereira de Andrade

A emergênCia do direito transnacional ambiental .............................................18 Priscila Pereira de Andrade

Desafíos y RESPUESTAS TRANSNACIONALES FRENTE A LOS CRÍMENES AMBIENTALES ...............30 Rosmerlin Estupiñan-Silva

DiREITO TRANSNACIONAL E MUdANÇAS CLIMÁTICAS .50 Géraud de Lassus Saint-Geniès

Especies en movimiento: la Convención sobre el Comercio Internacional de Especies Amenazadas de Fauna y Flora Silvestres como espacio de “Encuentro” de discursos, ACTORES Y ESTRATEGIAS EN EL DERECHO AMBIENTAL TRASNACIONAL

María Valeria Berros e Dabel Leandro Franco

El carácter transnacional del Sistema comunitario de ECogestion « Eco-ManageMENT AND Audit SCHEME » (EMAS) DENTRo de LA UE y MÁs ALlÁ DE SUS Fronteras ......72 Adélie Pomade

O CONCEITO DE CONDUTA EMPRESARIAL RESPONSÁVEL À LUZ DOS ORDENAMENTOS JURÍDICOS BRASILEIRO, INTERNACIONAL E TRANSNACIONAL

Gabriel Webber Ziero

ARBITRAGEM NO DIREITO TRIBUTÁRIO INTERNACIONAL E NO DIREITO INTERNACIONAL DOS INVESTIMENTOS: UMA MANIFESTAÇÃO DO DIREITO TRANSNACIONAL

Vivian Daniele Rocha Gabriel 
O DIREITO TRIBUTÁRIO SOB UMA PERSPECTIVA TRANSNACIONAL

Franciele de Simas Estrela Borges

As Características do Direito Transnacional como Metodologia: Análise sob o enfoQue dos Aspectos Processuais da Arbitragem 126

Flávia Foz Mange

O DIREITO TRANSNACIONAL (“GLOBAL LAW") E A CRISE DE PARADIGMA DO ESTADO-CENTRISMO: É POSSÍVEL CONCEBER UMA ORDEM JURÍDICA TRANSNACIONAL? ...................................... 146

Luiza Nogueira Barbosa e Valesca Raizer Borges Moschen

TransPorte AÉREO E DIREITO TRANSNACIONAL: DA CONVERGÊNCIA À UNIFORMIDADE 160 Mickael R. Viglino

Outros Artigos. 175

O Fundo Monetário Internacional e a proteção dos direitos humanos: uma análise DO PROGRAMA DE CRESCIMENTO E REDUÇÃO DA POBREZA NO HAITI 177

Pablo Henrique Hubner de Lanna Costa e Carlos Alberto Simões de Tomaz

Um estranho no ninho? Padrões privados no Acordo de Barreiras Técnicas ao CoMÉRCIO DA OMC 192

Michelle Ratton Sanchez Badin e Marina Yoshimi Takitani

Os benefícios tributários do programa Inovar-Auto e os princípios da Nação Mais Favorecida e do Tratamento Nacional: uma análise dos argumentos dos Painéis atualmente em Curso contra o Brasil no Órgão de SoluÇão de Controvérsias da OMC . 211 Eric Moraes Castro e Silva

A ERA DA HUMANIDADE: REFLEXões PARA A HISTÓRIA DO DIREITO INTERNACIONAL 236 Henrique Weil Afonso

Precedentes vinculantes nos Estados Unidos da América e no direito brasileiro: Um ESTUDO COMPARADO 264

Patrícia Perrone Campos Mello 
IL DIRITTO AMBIENTALE SECONDO L'OTTICA DEL DIRITTO COSTITUZIONALE POSITIVO E LA RESPONSABILITÀ PER DANNI ALL'AMBIENTE NEL DIRITTO COMUNITARIO: LO STATO DELL'ARTE DEL DIRITTO AMBIENTALE COSTITUZIONALE E COMUNITARIO 287

Elcio Nacur Rezende

DA DESCONSIDERAÇÃo DA PERSONALIDADE JURÍDICA NAS RELAÇÕES CONSUMEIRISTAS BRASILEIRAS: ANÁLISE À LUZ DAS TEORIAS CLÁSSICAS

Daniel Amin Ferraz e Marcus Vinicius Silveira de Sá

ANALYSIS OF ADVANTAGES AND DISADVANTAGES OF FORUMS PRESCRIBED UNDER THE UNCLOS AND STATE PRACTICE: THE WAY AHEAD FOR INDIA ......................................................319

Vinai Kumar Singh

Do governo POR LEIS À governanÇA POR NúMERos: breve anÁlise do Trade in SERVICE AgreEMENT (TISA) ...............................................................................338 Jânia Maria Lopes Saldanha, Rafaela da Cruz Mello e Têmis Limberger

As DIRETIVAS EUROPEIAS COMO NORMA REGULADORA DO DIREITO ADMINISTRATIVO GLOBAL ..356 Alice Rocha da Silva e Ruth Maria Pereira dos Santos

O desenVolvimento da POlítica AGRícola COMUM dA UNião EUROPEIA 375 Tatiana de A. F. R. Cardoso Squeff

A imunidade de Jurisdição das organizaÇões internacionais FaCE AO Direito de aCESSO À JUSTIÇA 391

Fernanda Araújo Kallás e Caetano

O DIREITO INTERNACIONAL ENTRE O DEVER ÉTICO E A AÇÃo POLÍ́TICA: OS FUNDAMENTOS DE UM DEVER DE COOPERAÇÃO INTERNACIONAL NA FILOSOFIA POLÍTICA DE IMMANUEL KANT .405 Ademar Junior Pozzatti

EXTENSÃo E FRAGMENTAÇÃo NO CONTEXTO DA JURISDIÇÃO PENAL INTERNACIONAL .423 Marcus Vinícius Xavier de Oliveira

A DEFINiÇÃo JURÍdiCA DA "COMUNIDADE" .444 Nitish Monebhurrun, Michelle Lucas Cardoso Balbino, Fernanda Castelo Branco Araujo, Othon Pantoja, Míara Bogo Bruno e Cândida Dettenborn Nóbrega 
Comparative Study on Chinese Local Legislation of Science and Technology ProGRESS

LI Xiaoming e LI Yihan

O CONTROLE PENAL DO TRÁFICO DE PESSOAS: CONSTRUÇÃO JURÍDICA, INTERAÇÕES ORGANIZACIONAIS E COOPERAÇÃO INTERNACIONAL

Bruno Amaral Machado e Priscilla Brito Silva Vieira

Desativismo judicial: a extradição Battisti no Supremo Tribunal Federal .505 Francisco Rezek e Israel Paulino

A decisão norte-americana do Caso Myriad: novos paradigmas para a Proteção patenTÁRIA DO CÓDIGO GENÉTICO HUMANO E BIOTECNOLOGIA 514 José Carlos Vaz e Dias e Clarisse De La Cerda 


\section{Especies en movimiento: la Convención sobre el Comercio Internacional de Especies Amenazadas de Fauna y Flora Silvestres como espacio de "encuentro" de discursos, actores y estrategias en el derecho ambiental trasnacional*}

\author{
Species in motion: the Convention on \\ International Trade in Endangered Species \\ of Wild Fauna and Flora as a space for \\ articulating discourses, actors and strategies \\ in transnational environmental law.
}

\author{
María Valeria Berros** \\ Dabel Leandro Franco***
}

\section{Resumen}

En este artículo se propone mostrar la tendencia a la articulación del discurso jurídico con otros discursos provenientes, a su vez, de diferentes organismos e instituciones, que dejan huellas visibles en la configuración de normas jurídicas referidas a alguno de los aspectos que integran la cuestión ecológica. Desde esta perspectiva, este trabajo posee como punto de partida un análisis de caso: la Convención sobre el Comercio Internacional de Especies Amenazadas de Fauna y Flora Silvestres, la que, desde una estrategia de regulación del comercio, se intenta consolidar como un sistema de protección de la flora y de la fauna basado en la idea de que el comercio de vida silvestre sería controlado por listas de especies amenazadas preparadas y actualizadas continuamente. Junto con ese movimiento de actualización contínuo se tornan visibles las articulaciones de actores, instituciones y discursos heterogéneos, entre los cuales asume un rol preponderante el discurso científico-técnico pero, también, aparecen con carácter relevante los discursos de organismos gubernamentales y no gubernamentales siempre que su capacidad técnica así lo amerite.

Palabras clave: Derecho Internacional Ambiental - CITES - Derecho Ambiental - Discursos expertos

\section{Abstract}

This contribution aims at present the articulation among the juridical discourse and other ones, from different organizations and institutions, in the regulations about different aspects of the ecological issues. From this perspective, this article develops a case study: the Convention on Interna- 
tional Trade in Endangered Species of Wild Fauna and Flora. This Convention organise the international trade of species as a way to protect flora and fauna based on the idea that the trade of species could be controlled by lists of endangered species, constantly actualized. In this process it is visible to identify the articulations between different actors, institutions, discourses, between which the scientific and technique ones appears with a central role. In parallel the discourses of governmental and non-governmental organizations with a technical capacity also have a relevant role.

Key Words: International Environmental Law - CITES - Environmental Law - Experts Discourses.

\section{INTRODUCCIÓN.}

¿Cuáles son los criterios a través de los que se determina que una especie se encuentra extinta, amenazada, vulnerable? Estos tres conceptos, entre otros, aluden a categorías que suelen permear las diversas regulaciones en materia de protección de especies. Regulaciones que, a su vez, dialogan entre diferentes espacios de producción normativa y una heterogeneidad de actores, discursos y estrategias que "se encuentran" y traducen en términos regulatorios.

Este artículo se propone focalizar en el último tema mencionado y mostrar la tendencia a la articulación del discurso jurídico con otros discursos provenientes de distintos organismos e instituciones que dejan huellas visibles en la configuración de normas referidas a algunos de los aspectos que integran la cuestión ecológica, en este caso, la protección de especies amenazadas. Allí se tornan observables insumos provenientes de discursos expertos pertenecientes al sistema científico u organizaciones no gubernamentales de naturaleza internacional, que se relacionan con el derecho y permean su contenido y puesta en funcionamiento. Ello, a su vez, genera que en los ámbitos nacionales, locales, regionales, se tomen en consideración estos criterios clasificatorios para la toma de decisiones sobre la protección de especies de flora o fauna mediante políticas de diferente tipo que pueden conducir a la implementación de pautas de comercialización como a la disposición de áreas protegidas o especies específicamente tuteladas, entre la diversidad de estrategias contemporáneas relativas a esta problemática.
Estas articulaciones entre diversos discursos y actores permiten ilustrar un proceso que ha adquirido renovada centralidad en el campo jurídico en los últimos años de cara a los problemas que trascienden las fronteras nacionales, lo que es claramente observable respecto de la cuestión ecológica. En este contexto, la pérdida de biodiversidad constituye uno de los grandes problemas a nivel mundial junto, verbigracia, con el cambio climático. ${ }^{1}$

La Evaluación de Ecosistemas del Milenio en 2005 alertó sobre el grave y declinante estado de la biodiversidad. $^{2}$ En similar sentido, la Perspectiva Mundial sobre Diversidad Biológica publicada en 2010 puntualiza que no se ha alcanzado la meta de reducción de pérdida de biodiversidad que se había comprometido en 2002 y, asimismo, afirma que ocho años más tarde se ha profundizado la situación de retroceso en numerosos casos. $^{3}$ En este escenario de gravedad y urgencia, se construye el Plan Estratégico para la Diversidad Biológica 2011-2020 y las metas de Aichi fijadas en la COP 10 de Nagoya en 2010, con lo que se busca alcanzar nuevos objetivos de protección en un horizonte temporal de corto-mediano plazo. ${ }^{4}$

Esta situación por la que atraviesan las especies, cabe señalar, ha sido objeto de múltiples regulaciones y planes además de los mencionados, incluso, varias décadas antes del fin de mileno. La Convención sobre el Comercio Internacional de Especies Amenazadas de Fauna y Flora Silvestres (en adelante CITES) de 1973 es una muestra de esta preocupación traducida al derecho internacional que, en paralelo, permea las regulaciones de otros espacios normativos. En esta convención, y desde una estrategia de regulación del comercio, ${ }^{5}$ se in-

1 No es casual que en 1992, año de realización de la Cumbre de Naciones Unidas sobre Medio Ambiente y Desarrollo que colocó el problema ambiental en un primer plano, se hubiere adoptado tanto el texto de la Convención sobre Diversidad Biológica como el de la Convención Marco sobre el Cambio Climático.

2 Consultar: http://www.unep.org/maweb/es/index.aspx

3 Informe Perspectiva Mundial sobre la Biodiversidad 3, Secretaría del Convenio sobre Diversidad Biológica. 2010. Disponible en: http:// www.cbd.int/doc/publications/gbo/gbo3-final-es.pdf Cabe destacar que el año 2010 había sido calificado como "Año Internacional de la Diversidad Biológica".

4 Las denominadas Metas de Aichi identifican cinco objetivos estratégicos y sus respectivas metas para el año 2020. Disponible en: http://www.cbd.int/sp/targets/

5 Sobre esta categoría se encuentra en desarrollo un proyecto de iniciación a la investigación en el marco del Proyecto de Investigación: "Codex humano: normas, tecnologías y programas para el gobierno de lo vivo" en la Universidad Nacional del Litoral lo que se traduce en recientes producciones: FRANCO, Dabel Leandro. 
tenta consolidar un determinado sistema de protección de la flora y de la fauna. Sistema que, también, conduce a que "el movimiento" entre los diferentes apéndices de la norma derive en un determinado tratamiento de una especie en la medida en que a través de ellos transita. ${ }^{6}$

La CITES se basa en la idea de que el comercio de vida silvestre sería controlado por listas de especies amenazadas preparadas y actualizadas de manera periódica y a las que corresponde una específica reglamentación. De este modo, la ubicación de las especies en cada uno de sus diferentes apéndices implica un régimen regulatorio específico. Este movimiento de actualización continuo que conduce, a su vez, a un tránsito de las especies entre los diferentes apéndices que estructuran la convención referida, permite construir una serie de interrogantes para desmontar las articulaciones de actores, instituciones y discursos heterogéneos que dan contenido a normativas de este tipo.

\section{Observar el campo jurídico desde el ENTRELAZAMIENTO DE DISCURSOS, ESTRATEGIAS Y ACTORES: UNA PERSPECTIVA POSIBLE.}

Existen diferentes maneras de analizar cómo se construye el derecho. Una de entre las posibles, desde una perspectiva socio-jurídica de análisis, consiste en desmontar la articulación de discursos provenientes de distintos ámbitos institucionales, expertos, etcétera, que, finalmente, son los que definen el contenido de las regulaciones. En numerosos supuestos, además, son recomendaciones o bien iniciativas de instituciones no estatales internacionales las que poseen un rol medular en la construcción normativa que, a posteriori, también termina por inspirar otras escalas regulatorias. ${ }^{7}$

En este último sentido, y dentro de la perspectiva que enfoca en la globalización del derecho, es posible identificar la circulación de categorías, conceptos, ma-

La cuestión animal: entre la regulación del comercio y la desmercantilización de lo vivo en: Revista Catalana de Dret Ambiental, VII(1), 2016, 1-29.

6 Un estudio de caso en relación a este tema desde el campo de los estudios sociales de la ciencia en: THOMPSON, Charis. Coproducing CITES and the African elephant en: Jasanoff, Sheila, States of knowledge. The co-production of science and social order. London: Routledge, 2004.

7 SANTOS, Boaventura de Sousa. A globalizacao e as ciencias sociais. Sao Paulo: Cortéz, 2002. neras de normar una situación que se replican y transi$\tan$ entre los diversos espacios regulatorios. Un aporte para pensar la circulación entre los diferentes espacios de producción normativa propone tres escalas - el derecho local, nacional y mundial - en una suerte de "cartografía simbólica del derecho". ${ }^{8}$ La diferenciación de tales escalas viabiliza el seguimiento del tránsito de categorías y conceptos a su interior. En otros términos y en relación al tema que nos ocupa: permite observar como la extinción, amenaza, vulnerabilidad, entre otras conceptualizaciones, a la vez que circundan el ámbito internacional, también fundamentan y son argumento de normas nacionales y locales que, verbigracia, en virtud del estado de extinción de una especie deciden implementar una determinada política de conservación que, luego, podría modificarse en razón de pasar la especie a ser calificada como amenazada o vulnerable.

Ambas miradas o perspectivas para el análisis socio-legal confluyen en este trabajo al momento de indagar en la CITES. Cabe destacar, más en general, que en el ámbito de los problemas ecológicos este tipo de circulación y articulaciones discursivas se torna visible en la medida en que, en numerosos casos, son anexos y apéndices técnicos - realizados o propuestos, en muchos casos, por heterogéneos actores - los que terminan por dar contenido a la regulación.

Observaciones de este tipo se han realizado en relación a las evaluaciones de impacto ambiental, el establecimiento de estándares de emisión, entre otros, que han conducido a afirmar la existencia de una deriva cientificista del derecho que, como una de sus manifestaciones, alude a la remisión por parte del derecho a las determinaciones de la ciencia. ${ }^{9}$ Por otra parte, una aproximación a este texto internacional permite también indagar cómo el contenido de esta norma jurídica y sus racionalidades subyacentes se trasladan del ámbito global hacia el nacional y local.

Desde esta mirada podría observarse la CITES que, en la medida en que se estructura a partir de una serie

8 "El derecho local es una legalidad a gran escala; el derecho nacional - estatal es una legalidad de escala media; y el derecho mundial es una legalidad de pequeña escala", SANTOS, Boaventura de Sousa. Crítica de la razón indolente: contra el desperdicio de la experiencia. Bilbao: Desclée de Brouwer, 2003. p. 234.

9 La otra vía que permite aludir a una deriva cientificista del derecho es la conquista por parte de la ciencia de espacios propios del derecho tradicionalmente bajo su dominio. Véase: PARDO, José Esteve. El desconcierto del Leviatán. Politica y derecho ante las incertidumbres de la ciencia. Madrid: Marcial Pons, 2009. Pág. 125. 
de Apéndices en los cuales se colocan y clasifican las diferentes especies de fauna y flora - asignándosele así un determinado tipo de regulación - permite indagar en el modo mediante el cual se configura y re-configura el funcionamiento de esta convención internacional y, de manera concomitante, como se modifican las condiciones de protección de especies conforme su movimiento entre los diferentes Apéndices.

Así, esta norma forma parte del conjunto de regulaciones ambientales que pueden ser leídas en esta clave en la que confluyen el discurso científico y otros discursos en la tarea de diseño de los marcos institucionales y las regulaciones procedimentales tendientes a normar el comercio con fines protectorios. Una presentación focalizada en el funcionamiento de CITES pero que permite la apertura de interrogantes y reflexiones más generales sigue a continuación.

\section{Estrategias de ARTICULACIÓN ENTRE DISCURSOS Y ACTORES EN LA ESTRUCTURA Y FUNCIONAMIENTO DE LA CITES.}

La CITES surge de la Conferencia de Plenipotenciarios para concluir una Convención Internacional sobre el Comercio de Ciertas Especies de Fauna y Flora Silvestre organizada por los Estados Unidos y realizada en el Pentágono, Washington, entre el 12 de febrero y el 2 de marzo de 1973. La misma contó con la presencia de representantes de 80 países en calidad de participantes, así como representantes de 8 países y 6 organizaciones internacionales en calidad de observadores. Finalizado el encuentro, el 3 de marzo de ese mismo año, se acordó la CITES con la firma de 21 de los 80 países que participaron, y entró en vigor el $1^{\text {ro }}$ de julio de 1975 tras la ratificación de diez Estados.

En el texto de la convención se estableció la Conferencia de las Partes como órgano de adopción de decisiones, a la cual se le atribuyen las facultades de formulación de recomendaciones y de enmienda de los Apéndices. Por su parte, se nombró a Suiza como Gobierno Depositario y se constituyó una Secretaría administrada por el Programa de las Naciones Unidas para el Medio Ambiente (PNUMA) encargada de organizar las reuniones, realizar estudios y preparar informes, entre otras atribuciones.
Conforme la "Visión Estratégica de la CITES: 20082020", su misión consiste en:

Conservar la diversidad biológica y contribuir a su utilización sostenible, velando por que ninguna especie de fauna o flora silvestres se someta o se siga sometiendo a una explotación insostenible debido al comercio internacional, contribuyendo así a una reducción considerable del índice de pérdida de biodiversidad y aportando una contribución significativa para lograr las Metas de Aichi para la Diversidad Biológica relevantes. ${ }^{10}$

Como se ha adelantado, la convención adoptó una estrategia de protección mediante la regulación del comercio, para lo cual se elaboraron listas de especies amenazadas, preparadas y actualizadas por expertos, que tomaron como antecedente la Lista Roja de Especies Amenazadas publicada por la Unión Internacional para la Conservación de la Naturaleza (en adelante UICN) desde el año $1963 .^{11}$

En consecuencia, las especies de fauna y flora fueron distribuidas en tres apéndices con diferentes niveles de protección, los cuales se encuentran caracterizados en el Artículo II de la convención:

(i) el Apéndice I incluye todas las especies en peligro de extinción que pueden ser afectadas por el comercio, el cual se encuentra prohibido salvo determinadas autorizaciones otorgadas en circunstancias excepcionales;

(ii) el Apéndice II incluye a las especies que no se encuentran necesariamente en peligro de extinción en la actualidad pero que podrían llegar a estarlo a menos que no se reglamente de forma estricta su comercio y, además, aquellas otras especies que, si bien no se encuentran afectadas por el comercio, deben reglamentarse con el objetivo de permitir un control más eficaz de las primeras;

(iii) el Apéndice III incluye a las especies que las Partes deseen proteger dentro de su jurisdicción y para lo cual necesitan de la cooperación de otros Estados.

A su vez, el comercio de las especies incluidas en cada Apéndice fue regulado en relación a: exportación, importación, reexportación e introducción proveniente del mar.

10 CITES. Resolución Conf. 16.3, 2013.

11 KAKABADSE, Yolanda. "Carta Magna para la vida silvestre" en: El comercio de especies. La CITES en el nuevo milenio, Conservación Mundial, Boletín de la UICN, 33(3), 2002. 
Del texto de la convención surgen dos articulaciones. En primer lugar, entre el discurso jurídico y el discurso experto científico-técnico. En segundo lugar, entre el ámbito regulatorio y el discurso experto proveniente de organizaciones no gubernamentales dedicadas a la protección de especies o, más ampliamente, a la conservación de la naturaleza, lo que se puede incluso relacionar con el momento inicial de la CITES en el que tuvieron un anclaje importante las listas de especies amenazadas confeccionadas por UICN.

Con respecto a la primera articulación, la reglamentación del comercio que propone la CITES se sustenta en una serie de "permisos y certificados", los cuales deben ser concedidos en cumplimiento de un conjunto de requisitos que involucran la intervención de autoridades tanto "administrativas" como "científicas". ${ }^{12}$

Estas autoridades se encuentran reguladas en el Artículo IX de la CITES y son designadas por las partes. Las Autoridades Administrativas tienen a su cargo "conceder permisos o certificados" en nombre de la Parte que la designó, ocupando un rol fundamental en la regulación del comercio propuesta por la convención. Por su parte, el rol de las Autoridades Científicas depende del grado de protección asignado a cada especie.

Para los especímenes del Apéndice I se requiere la constatación, por parte de la Autoridad Científica del Estado de introducción, de que la exportación, importación o introducción procedente del mar "no perjudicará la supervivencia de la especie". ${ }^{13}$

En la reglamentación del comercio de especímenes de especies incluidas en el Apéndice II encontramos este mismo requisito en relación a los permisos de exportación y a los certificados para introducción procedente del mar, con un postulado llamativo. Luego de someter los permisos de exportación al control de una Autoridad Científica de cada parte se establece que:

Cuando una Autoridad Científica determine que la exportación de especímenes de cualquiera de esas especies debe limitarse a fin de conservarla, a través de su hábitat,

12 La distinción entre la "ladera científica" y la "ladera política" en los procesos de toma de decisiones por parte del Estado para la gestión de riesgos de la jurista francesa, Christine Noiville, se convierte aquí en un insumo interesante para pensar esta doble intervención científico y administrativa que propone CITES. NOIVILLE, Christine, Du bon gouvernement des risques. Le droit et la question du « risque acceptable ». Paris : Presses Universitaires de France, 2003.

13 CITES, Artículo III. en un nivel consistente con su papel en los ecosistemas donde se halla y en un nivel suficientemente superior a aquel en el cual esa especie sería susceptible de inclusión en el Apéndice I, la Autoridad Científica comunicará a la Autoridad Administrativa competente las medidas apropiadas a tomarse, a fin de limitar la concesión de permisos de exportación para especímenes de dicha especie. ${ }^{14}$

Sobre este apartado, se ha afirmado que, si bien la Autoridad Científica debe informarse respecto de los efectos del comercio de las especies de su país, muchos países exportadores carecen de "datos científicos" suficientes para determinar el estado de sus poblaciones así como los efectos que la comercialización produce sobre las mismas, lo que permite convalidar el carácter técnico de la fundamentación de la decisión de proteger. ${ }^{15}$

Respecto del comercio de especímenes de especies incluidas en el Apéndice III, las Autoridades Científicas no se encuentran contempladas en la regulación, sometiéndose el mismo al control de las Autoridades Administrativas. Cabe tener en cuenta que, a su vez y conforme lo establece el Artículo II, el Apéndice III incluye aquellas especies que se encuentran reglamentadas dentro de la jurisdicción de cualquiera de las Partes y estas precisen de la colaboración de otras Partes en el control de su comercialización. Este apartado se diferencia de los Apéndices I y II que refieren al concepto de "peligro de extinción" - especies efectivamente en peligro de extinción, en el Apéndice I, y especies potencialmente en peligros de extinción en el Apéndice II -, el cual remite a su vez al discurso científico-técnico.

De esta manera, podemos constatar dos discursos que "fundamentan" la protección de especies: el discurso científico-técnico, que adquiere relevancia en relación a los Apéndices I y II, y el discurso de la Autoridad Administrativa, que justifica la protección de las especies del Apéndice III. A su vez, de la mayor participación de las Autoridades Científicas en la reglamentación del comercio de especies incluidas en el Apéndice I respecto del comercio de especies del Apéndice II, surge que el discurso científico-técnico adquiere mayor relevancia cuanto mayor sea la amenaza de extinción.

En el esquema de la CITES, el discurso científico-técnico también se visibiliza en las funciones de la Secretaría, entre las que se identifica "realizar estudios

14 CITES, Artículo IV.

15 Wijnstekers, Willem. La evolución de la CITES. Secretaría de la CITES, 2003. 
científicos y técnicos (...) incluyendo estudios relacionados con normas para la adecuada preparación y embarque de especímenes vivos y los medios para su identificación" y "formular recomendaciones (...) incluyendo el intercambio de información de naturaleza científica o técnica". ${ }^{16}$ Estos postulados se caracterizan por presentar afirmaciones normativas como afirmaciones científicas y afirmaciones científicas como normativas $^{17}$, las cuales se corresponden, a su vez, con actores estatales, sociales y científicos.

Asimismo, en los apartados referidos a los procesos de enmienda de los Apéndices se encuentran postulados que denotan el carácter técnico-científico que debe fundamentar la movilidad de especies entre los mismos. Por ej., en relación a las propuestas de enmienda a los Apéndices I y II referidas a especies marinas, se obliga a la Secretaría a consultar "con las entidades intergubernamentales que tuvieren una función en relación con dichas especies, especialmente con el fin de obtener cualquier información científica que éstas puedan suministrar". ${ }^{18}$ Por su parte, se establece que las Partes pueden "trasmitir a la Secretaría sus comentarios sobre la enmienda propuesta, junto con todos los datos científicos e información pertinentes". ${ }^{19}$

Sin embargo, los postulados referidos al proceso de aprobación de las enmiendas presentan un carácter más político. En los Apéndices I y II encontramos dos supuestos:

(i) si las propuestas de enmienda fueron presentadas en reuniones de la Conferencia de Partes, serán adoptadas por una "mayoría de dos tercios de las Partes presentes y votantes";

(ii) si fueron presentadas entre reuniones de la Conferencia de Partes, hay dos posibilidades: si no recibe objeción, entrará en vigor de manera automática y, si recibe objeción, la enmienda propuesta será puesta a votación por correspondencia.

Por último, cabe señalar que en el proceso de enmienda del Apéndice III predomina el carácter político y la decisión toma una raigambre más discrecional. En tal sentido, se estima que "[c]ualquier parte podrá, en cualquier momento, enviar a la Secretaría una lista de especies que manifieste se hallan sometidas a regla-

16 CITES, Artículo XII.

17 SANTOS, Boaventura de Sousa. Crítica de la razón indolente: contra el desperdicio de la experiencia. Bilbao: Desclée de Brouwer, 2003. Pág. 57.

18 CITES, Artículo XV, inc. 2., a).

19 CITES, Artículo XV, inc. 2., d). mentación dentro de su jurisdicción", ${ }^{20}$ las cuales serán incluidas en el Apéndice III. No se requiere aceptación de las Partes, sino sólo de la notificación, conservando las Partes el derecho a hacer reservas respecto de la especie en cuestión. Luego se agrega que la Parte "que envíe una lista de especies para inclusión en el Apéndice III, podrá retirar cualquier especie de dicha lista en cualquier momento, mediante notificación a la Secretaría, la cual comunicará dicho retiro a todas las Partes". ${ }^{21}$

En este caso la ciencia no actúa como condicionante de la política, sino que le otorga un amplio margen de acción ya que, al no entrar en juego conceptos que remiten necesariamente al conocimiento científico - por ej., "especies amenazadas" -, el proceso de enmienda se reduce a una mera decisión institucional. Como correlato de esto, la Conferencia de Partes ha rechazado la incorporación de especies que no estaban amenazadas por el comercio internacional ${ }^{22} \mathrm{y}$ ha intentado brindarle un carácter más "científico" a la incorporación de estas en el Apéndice III mediante la Resolución Conf. 8.23, en la cual se recomienda que "las Partes soliciten asesoramiento al Comité de Fauna o al Comité de Flora sobre los datos biológicos y de índole comercial sobre esa especie" y que "las Partes que hayan incluido especies en el Apéndice III examinen detenidamente la situación de esas especies y la conveniencia de mantenerlas en él".

Una segunda cuestión a destacar es la interrelación que se plantea en CITES con las organizaciones no gubernamentales así como, también, con organismos de naturaleza gubernamental, la cual se encuentra supeditada a su capacidad técnica. De esta manera, se asocia aquí la relevancia de este tipo de discurso experto que, aún no procedente del ámbito científico institucionalizado, se relaciona con el mismo de diferente modo - ya sea a través de la formación de comisiones de expertos de diversos países, publicación de informes, reuniones y conferencias internacionales, etc. - y termina por traducirse en insumos propios de la organización.

En la "estructura" de la CITES, es decir, el articulado correspondiente a los órganos de la convención - Conferencia de las Partes y Secretaría - se le otorgan ciertas atribuciones - por ejemplo, de participar sin voto en la Conferencia de las Partes - a organismos o entidades gubernamentales o no gubernamentales, sean

20 CITES, Artículo XVI, 1.

21 CITES, Artículo XVI, 3.

22 Wijnstekersxz, Op. Cit. 
nacionales o internacionales, siempre y cuando posean "competencia técnica en la protección, conservación y administración de la fauna y flora silvestre". ${ }^{23}$

Del análisis de los párrafos precedentes, se puede observar en este sistema normativo para la protección de especies de flora y fauna una suerte de disociación entre la fundamentación, de carácter científico, y la decisión, de carácter político, a la vez que se verifican ciertos matices y desplazamientos en las diversas hipótesis sobre las que se desarrolla esta regulación. Regulación que, a su vez, ha inspirado normas de otras escalas normativas, ya sea regionales, nacionales o aún locales en las que las categorías y conceptos aquí establecidos cumplen un rol cardinal para la confección de políticas y normas relativas a este aspecto del problema ecológico.

\section{Reflexiones finales.}

El análisis de esta convención en particular se configura como una estrategia que conduce a introducir y desmontar un tema más general que se desarrolla al interior del campo jurídico y está dado por el entrelazamiento entre discursos y la circulación de conceptos, a partir de lo cual es observable cómo categorías y decisiones regulatorias transitan entre el espacio global y las demás escalas regulatorias.

Lo expuesto se torna aún más visible en el caso de la cuestión ecológica que, a la vez que se plantea como una problemática que claramente excede las fronteras nacionales, se asume como un tema que requiere de una fuerte presencia del discurso experto. En tal sentido, el discurso científico-técnico asume un rol central pero, también, aparecen con carácter relevante los discursos de organismos gubernamentales y no gubernamentales siempre que su capacidad técnica así lo amerite. Ambos planos permiten, entonces, observar la articulación entre discurso jurídico y experto de distinta naturaleza.

A modo de cierre, corresponde preguntarse qué otras aperturas posibles serían necesario consolidar en la articulación de discursos que, en el momento contemporáneo y desde otras estrategias y cosmovisiones, argumentan en torno a cómo mejorar el estado de la diversidad biológica y detener la extinción de especies. En ese sentido, la revalorización de otras formas de conocimiento que circulan

23 CITES, Artículos XI y XII. por diferentes ámbitos adquiere especial relevancia, así como las alternativas que se plantean, respecto de este tema, a la regulación del comercio desde las propuestas de desmercantilización de lo vivo ${ }^{24}$.

\section{Bibliografía}

FRANCO, Dabel Leandro. La cuestión animal: entre la regulación del comercio y la desmercantilización de lo vivo en: Revista Catalana de Dret Ambiental, VII(1), 2016, 1-29.

HOUTART, François. El Concepto de Sumak Kawsay y su Correspondencia con el Bien Común de la Humanidad en: América Latina en Movimiento, 2011, 1-20.

KAKABADSE, Yolanda. Carta Magna para la vida silvestre en: El comercio de especies. La CITES en el nuevo milenio, Conservación Mundial, Boletín de la UICN, 33(3), 2002.

NOIVILLE, Christine, Du bon gowvernement des risques. Le droit et la question $d u$ « risque acceptable ». Paris: Presses Universitaires de France, 2003.

PARDO, José Esteve. El desconcierto del Leviatán. Política y derecho ante las incertidumbres de la ciencia. Madrid: Marcial Pons, 2009.

SANTOS, Boaventura de Sousa. A globalizacao e as ciencias sociais. Sao Paulo: Cortéz, 2002.

SANTOS, Boaventura de Sousa. Crítica de la razón indolente: contra el desperdicio de la experiencia. Bilbao: Desclée de Brouwer, 2003.

THOMPSON, Charis. Co-producing CITES and the African elephant en: Jasanoff, Sheila, States of knowledge. The co-production of science and social order. Lon-

24 Los procesos de desmercantilización parten de una crítica al pensamiento moderno que rechaza, primordialmente, el modelo de desarrollo hegemónico, el carácter totalizador de la ciencia moderna, la concepción atomizada y desarticulada de la naturaleza, la visión antropocéntrica e utilitarista del desarrollo y la lógica del sistema capitalista de transformarlo todo en mercancía, y se orientan, a su vez, a reducir la esfera del mercado, promoviendo otras formas de relación social y otras maneras de hacer frente a las necesidades humanas y no humanas. Un abordaje de la cuestión en relación a las reformas legislativas que se produjeron en Latinoamérica - Ecuador y Bolivia - puede verse en: HOUTART, François. El Concepto de Sumak Kawsay y su Correspondencia con el Bien Común de la Humanidad en: América Latina en Movimiento, 2011, 1-20 y UNCETA, Koldo. Poscrecimiento, desmercantilización y «buen vivin» en: Nueva Sociedad, (252), 2014, 136-152. 
don: Routledge, 2004.

UNCETA, Koldo. Poscrecimiento, desmercantilización y «buen vivin» en: Nueva Sociedad, (252), 2014, 136-152.

WIJNSTEKERS, Willem. La evolución de la CITES. Secretaría de la CITES, 2003.

\section{NORMATIVAS, INFORMES Y DOCUMENTOS CONSULTADOS}

Convención sobre el Comercio Internacional de Especies Amenazadas de Fauna y Flora Silvestres, 1973.
Convención sobre Diversidad Biológica, 1992.

Convención Marco sobre el Cambio Climático, 1992.

Informe Perspectiva Mundial sobre la Biodiversidad 3, Secretaría del Convenio sobre Diversidad Biológica. 2010. Disponible en: http://www.cbd.int/doc/publications/ gbo/gbo3-final-es.pdf

Metas de Aichi. Disponible en: http://www.cbd.int/ $\mathrm{sp} /$ targets/ 
Para publicar na Revista de Direito Internacional, acesse o endereço eletrônico www.rdi.uniceub.br ou www.brazilianjournal.org.

Observe as normas de publicação, para facilitar e agilizar o trabalho de edição. 